\title{
Expression of CD73 and CD39 on Peripheral Blood Leukocytes in Type II Diabetes Patients
}

\author{
Soha Ezz El-Arab Abdel Wahab ${ }^{1}$, Deena Samir Eissa ${ }^{1}$, Mona Mohamed Abdelsalam ${ }^{2}$, \\ Christine Atef Habib ${ }^{1}$ \\ Departments of ${ }^{1}$ Clinical Pathology and ${ }^{2}$ Endocrinology \& Metabolism, Faculty of Medicine, Ain Shams University \\ Corresponding author: Soha Ezz El-Arab Abdel Wahab, Phone: 01006619021
}

\begin{abstract}
Background: Type II diabetes mellitus (T2D) is one of the most common chronic metabolic diseases. It is associated with a state of chronic inflammation resulting in both microvascular and macrovascular complications. The adinosinergic axis plays an important anti-inflammatory protective role through conversion of ATP (produced as a result of inflammation) into adenosine (a potent anti-inflammatory mediator) through CD39 and CD73. Various peripheral blood leucocytes hold CD39 and CD73 with different degrees.

Objective: To determine the expression of CD73 and CD39 on different peripheral blood leukocytes including Treg cells to uncover their inflammatory modulation role in T2D subjects in comparison to normal control ones. The level of their expression will be correlated with various anthropometric and biochemical parameters in diabetic patients.

Materials and Methods: Venous blood was collected on EDTA anticoagulated tubes from 45 T2D patients; 15 with early T2D and 30 with complicated T2D with both microvascular and macrovascular complications. Twenty-five normal subjects, with no family history of T2D, were included. Immunophenotyping of the samples were performed on Navios Flow cytometer (Coulter Electronics, USA), using phycoerythrin (PE)labelled CD39 antibodies, fluorescein isothiocyanate (FITC)-labelled CD4 antibodies, peridinin-chlorophyllprotein complex: CY5.5 conjugate (PerCP-CY5.5)-labelled CD73 and allophycocyanin conjugate (APC)labelled CD25.

Results: CD39 expression was highest in early cases of T2D in comparison to least expression in complicated T2D on peripheral blood total lymphocytes, CD4+ lymphocytes and CD4+CD25+ lymphocytes.

Conclusions: The increased expression of CD39 in early non-complicated cases of T2D indicates a state of low-level chronic inflammation owing to their role in production of adenosine; a potent anti-inflammatory mediator. This is reversed when CD39 expression in peripheral blood leucocytes is assessed in complicated T2D patients, which emphasise their role in protecting the patients from diabetic complications.
\end{abstract}

Keywords: Adenosine, CD39, CD73, diabetes, inflammation.

\section{INTRODUCTION}

Diabetes mellitus is a disorder of glucose metabolism characterized by a state of chronic hyperglycemia. It is caused by deficiency in insulin secretion, abnormal insulin action or both. It is a systemic disorder causing damage and dysfunction of multiple organs ${ }^{(\mathbf{1})}$.

There are two main pathological abnormalities in these patients. First, the insulin resistance which is a decline in the effect of insulin on the peripheral tissue, which is believed to be the primarily defect in T2D. Second, beta cell dysfunction which is a decrease in insulin synthesis in the pancrease making it unable to overcome the peripheral insulin resistance, thus there is a relative deficiency of insulin early in the disease and absolute insulin deficiency later on ${ }^{(2)}$. T2D is a multifactorial disorder in which both insulin resistance and beta cell dysfunction are influenced by multiple environmental and genetic factors ${ }^{(3)}$.

Chronic low-grade inflammation and activation of the immune cells are main factors in the pathogenesis of obesity-induced insulin resistance, the metabolic syndrome and T2D. In fact, in T2D there is infiltration of the liver, muscle, pancreas and adipose tissue by macrophages and other immune cells with switch of the profiles of these cells from an antiinflammatory phenotype to a pro-inflammatory phenotype. The pro-inflammatory phenotype contributes to the pathogenesis of T2D by producing cytokines that causes inhibition of insulin signalling in the peripheral tissues and induction of $\beta$-cell dysfunction ${ }^{(4)}$.

Diabetes mellitus is complicated by both acute and chronic complications. Chronic complications of diabetes can be both microvascular (retinopathy, nephropathy and neuropathy) and macrovascular (atherosclerosis, coronary artery disease, diabetic foot and stroke) complications.

Extracellular nucleotides, such as ATP, are important signalling molecules involved in many biological processes. Under basal conditions 
extracellular concentrations of ATP are maintained at low levels. Endogenous regulation of ATP concentration is mediated by CD39 and CD73, which hydrolyze ATP to adenosine which is a potent anti-inflammatory mediator. Both CD39 and CD73 expressions are dynamic and change under pathophysiological conditions (Fig. 1).

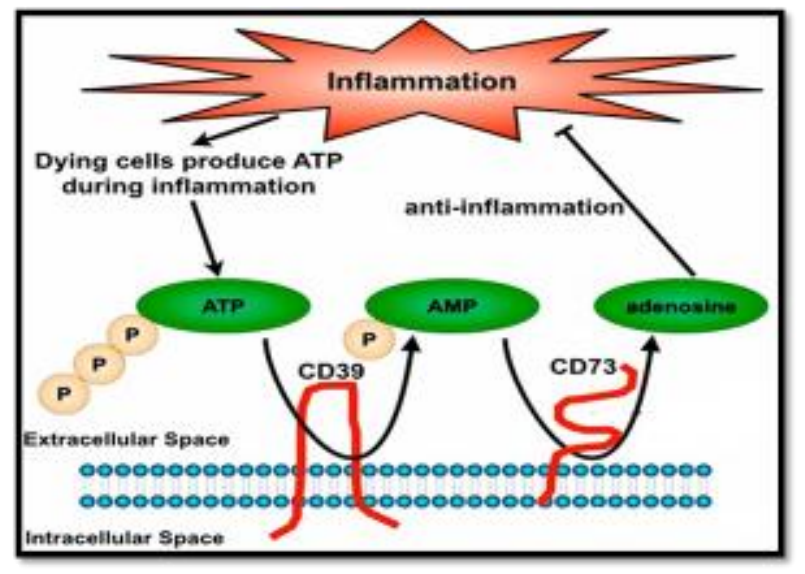

Fig. (1): Cell-mediated anti-inflammation through CD73/ adenosine ${ }^{(5)}$.

\section{AIM OF THE WORK}

This work aims to determine the expression of CD73 and CD39 on different peripheral blood leukocytes including Treg cells to uncover their inflammatory modulation role in T2D subjects in comparison to normal control ones. The level of their expression will be correlated with various anthropometric and biochemical parameters in diabetic patients.

\section{PATIENTS AND METHODS}

This study was conducted on 45 patients with T2D recruited from outpatient clinics of Ain Shams University Hospitals. They were 24 males and 21 females (M: F was 1.14:1), their ages ranged from 33 to 74 years with (Median [IQR], 57 [53.5-65]).

They were further divided into: Fifteen patients with uncomplicated, recently diagnosed ( $<5$ years duration) T2D. They were 7 males and 8 females (M: F was 1:1.14) whose ages ranged from 33 to 72 years (Median [IQR], 55 [53-70]). Thirty patients with complicated T2D; 15 patients with macrovascular complications (myocardial infarctions, strokes and diabetic foot) and 15 patients microvascular with complications (retinopathy, nephropathy and neuropathy) who were 17 male and 13 female with M:F was 1.3:1. Their ages ranged from 49 to 74 years (Median [IQR], 59.5 [56.5-64.5]).

A control group of 25 age and sex matched healthy adults, with no family history of diabetes, was included. They were 9 male and 16 female with M:F 1:1.77. Their ages ranged from 24 to 49 years (Median [IQR], 55 [28-38]).

Sampling: Two milliliters of venous blood were collected on EDTA $\left(\mathrm{K}_{3}\right.$ EDTA) anticoagulated tube (with concentration of $1.2 \mathrm{mg}$ of the anhydrous salt per $\mathrm{ml}$ of blood) for immunophenotyping.

Laboratory tests: Immunophenotyping of peripheral blood (PB) samples were performed on Navios Flow cytometer (Coulter Electronics,USA), using phycoerythrin (PE)-labelled CD39 antibodies, fluorescein isothiocyanate (FITC)labelled CD4 antibodies, peridinin-chlorophyllprotein complex: CY5.5 conjugate (PerCP-CY5.5)labelled CD73 and allophycocyanin conjugate (APC)-labelled CD25.

Statistical methods: IBM SPSS statistics (V. 24.0, IBM Corp., USA, 2016) was used for data analysis. Date was expressed as median and percentiles for quantitative non-parametric measures. The following tests were done: Comparison between two independent groups for non-parametric data using Wilcoxon Rank Sum test. Comparison between more than 2 patient groups for non-parametric data using Kruskall Wallis test. Ranked Sperman correlation test to study the possible association between each two variables among each group for non-parameteric data. The probability of error at 0.05 was considered significant, while at 0.01 and 0.001 were considered highly significant.

Ethical and approval statements: The study was approved by the Ethics Board of Ain Shams University and an informed written consent was taken from each participant in the study.

\section{RESULTS}

This study was conducted on 45 T2D patients. They were 24 male and 21 female, with M: F ratio 1.14:1. Their ages ranged from 33 to 74 years with (Median [IQR], 57 [53.5-65]). A control group of 25 age and sex matched healthy adults, with no family history of diabetes, was included. They were 9 male and 16 female with M: F 1:1.77. 
Their ages ranged from 24 to 49 years (Median [IQR], 55 [28-38]).

On total lymphocytes, CD39 expression was highest in the early cases and lowest in complicated cases $(p<0.05)$. In contrast, CD73 expression was comparable among early and complicated cases, and healthy controls ( $p>0.05$ ). Co-expression of CD73 and CD39 was higher in early cases and lower in complicated cases $(\mathrm{p}<0.05)$. Absence of both CD39 and CD73 was significantly detected in complicated cases $(\mathrm{P}=0)$ (Table 1, Fig. 2).

Table (1): Comparison of CD39 and CD73 expression on peripheral blood lymphocytes among healthy subjects, early and complicated T2D patients.

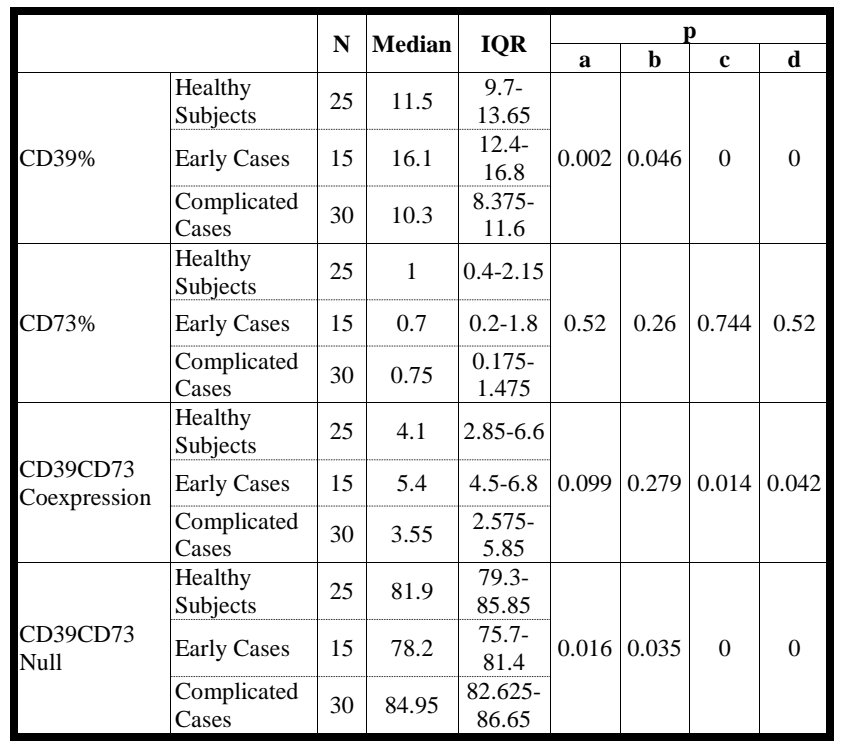

$\mathrm{a}=$ Control Vs Early, $\mathrm{b}=$ Control Vs Complicated, c= Early Vs Complicated, $\mathrm{d}=$ Control Vs Early Vs Complicated, $\mathrm{n}=$ Number, $\mathrm{IQR}=$ Interquartile Range, $\mathrm{p}=$ Probability of Error.

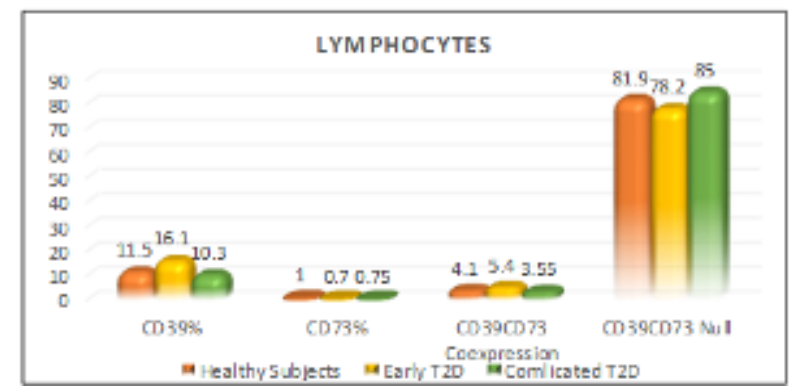

Fig. (2): Comparison of CD39 and CD73 expression on peripheral blood lymphocytes among healthy subjects, early and complicated T2D patients.

Lymphocytes were also gated based on their expression of CD4, where CD4+ cells showed high expression of CD39 in early cases in comparison to lower expression in healthy subjects and complicated cases $(\mathrm{p}<0.05)$. On the other hand, CD73 showed similar expression among the three groups ( $p>0.05)$. Co-expression of CD39 and CD73 was highest in early cases, moderate in healthy subjects and lowest in complicated cases ( $\mathrm{p}$ $<0.05)$. The absence of the two markers was significantly detected in the complicated cases (p $<0.05$ ) (Table 2, Fig. $3 \& 4$ ).

Table (2): Comparison of CD39 and CD73 expression on peripheral blood CD4+ Lymphocytes among healthy subjects, early and complicated T2D patients.

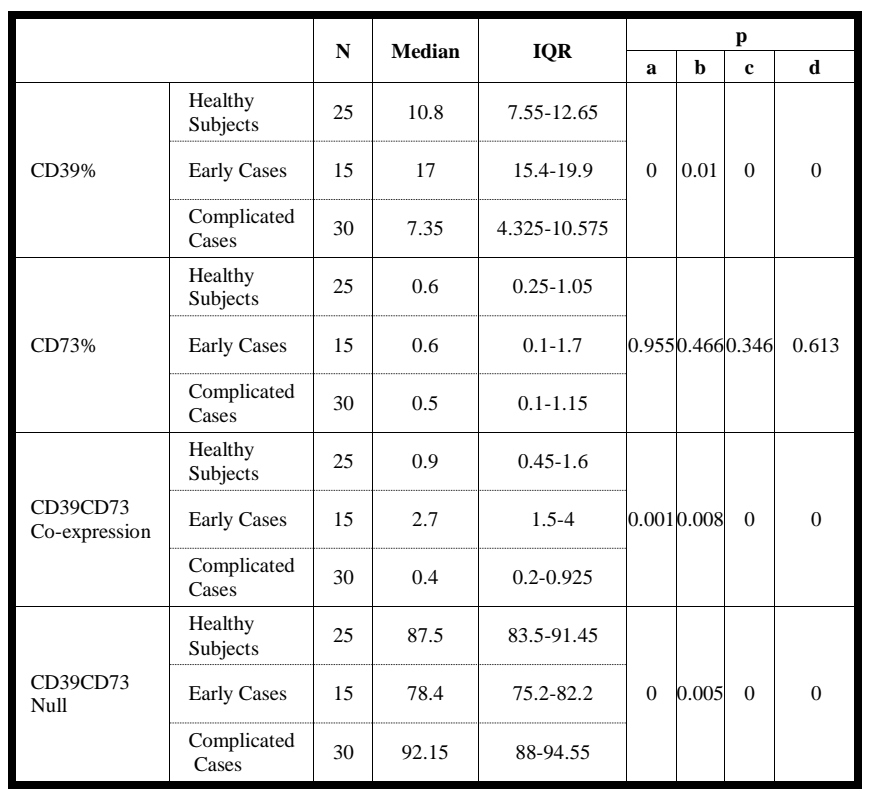

$\mathrm{a}=$ Control Vs Early, $\mathrm{b}=$ Control Vs Complicated, c= Early Vs Complicated, $d=$ Control Vs Early Vs Complicated, $n=$ Number, IQR=Interquartile Range, $\mathrm{p}=$ Probability of Error.

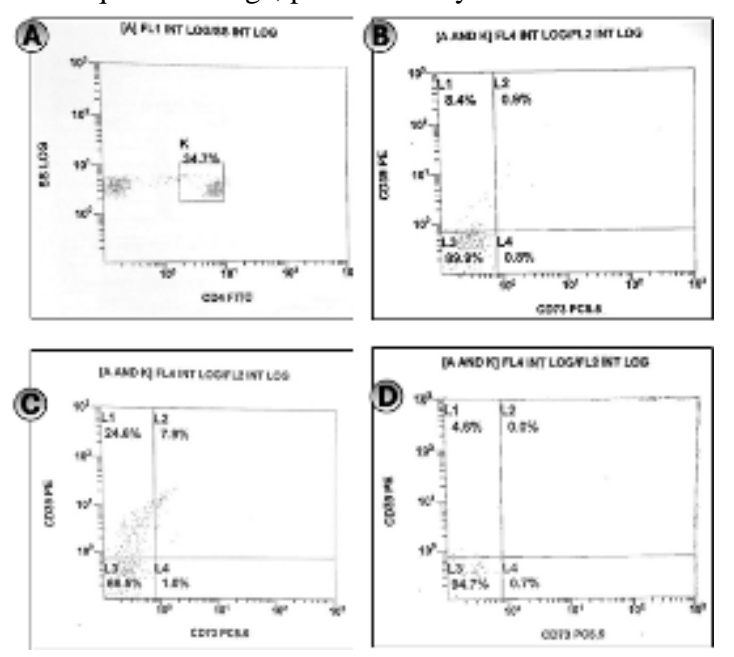

Fig. (3): Flowcytometry graph for (A) Lymphocytes gating based on expression of CD4,(B) CD39 and CD73 expression on CD4+ lymphocytes in Control subject, (C) Early T2D and (D) Complicated T2D patients. 


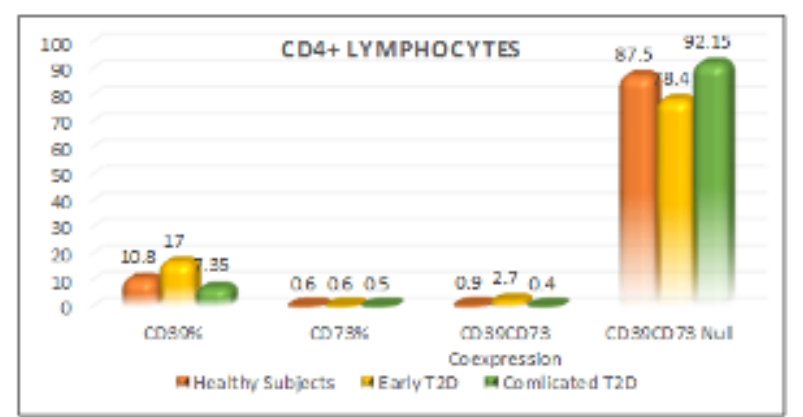

Fig. (4): Comparison of CD39 and CD73 expression on peripheral blood CD4+ Lymphocytes among healthy subjects, early and complicated T2D patients.

As recent evidence has shown the involvement of CD39 and CD73 in the functional role of Treg, CD4+ lymphocytes were further gated based on the degree of expression of CD25 giving rise to high, moderate and low expression of CD25 cells.

Expression of CD39 in CD4+CD25+Low, CD4+CD25+Moderate and CD4+CD25+ High Treg cells was high in the early diabetic patients versus low expression in the complicated diabetic patients ( $p$ $<0.05)$. On the contrary, CD73 expression on these cells was similar among the three groups $(\mathrm{p}>0.05)$. Co-expression of CD39 and CD73 was significally high in early and complicated T2D patients in all three subpopulations of CD4+CD25+ cells ( $p<0.05$ ). Absence of the two markers was noticed in the complicated cases in comparison to early T2D patients and healthy subjects $(\mathrm{p}<0.05)$ (Table 3 , Fig. $5-8)$.

Table 3: Comparison of CD39 and CD73 expression on peripheral blood CD4+CD25+ (Low, Moderate and High) Tregs among healthy subjects, early and complicated T2D patients

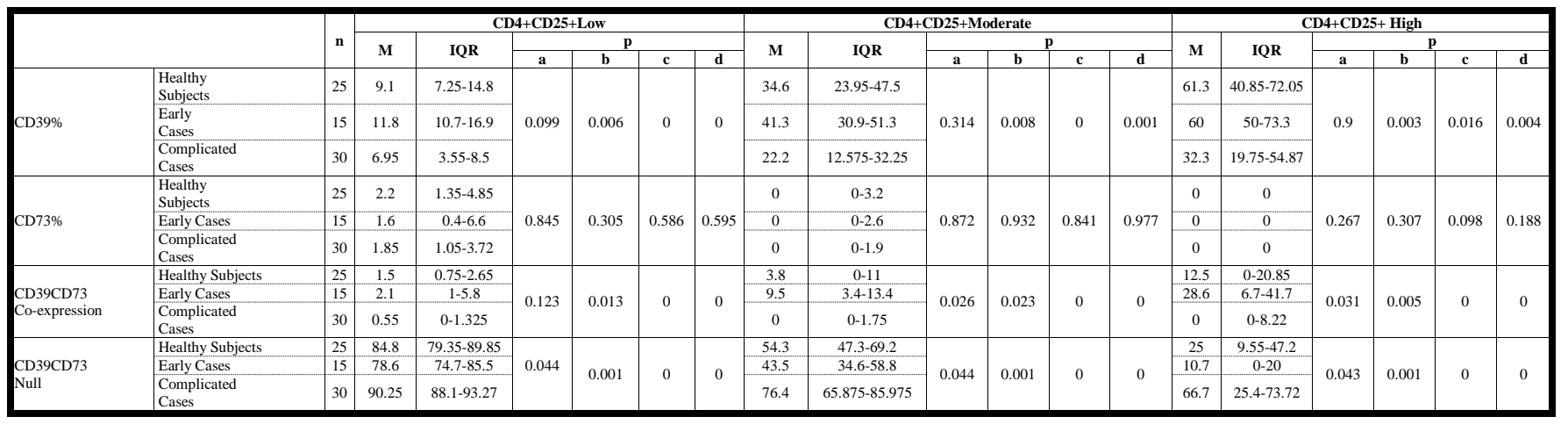

$\mathrm{a}=$ Control Vs Early, $\mathrm{b}=$ Control Vs Complicated, $\mathrm{c}=$ Early Vs Complicated, $\mathrm{d}=$ Control Vs Early Vs Complicated, $\mathrm{n}=$ Number, $\mathrm{IQR}=$ Interquartile Range, $\mathrm{p}=$ Probability of Error.
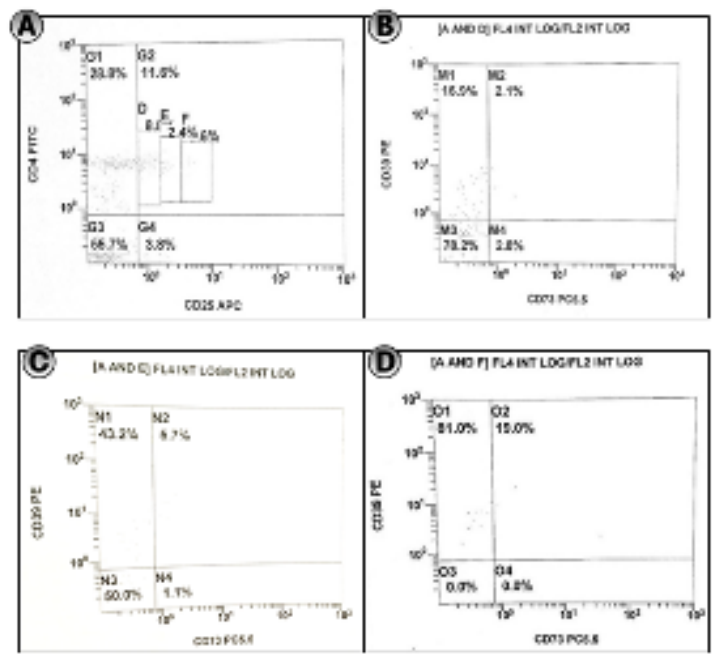

Fig. (5): Flowcytometry graph for (A) CD4+ lymphocytes gating based on degree of expression of $\mathrm{CD} 25$ giving rise to low $[\mathrm{D}]$, moderate $[\mathrm{E}]$ and high [F] expression of CD25 cells. (B) flowcytometry graph for CD39 and CD73 expression on CD4+CD25+Low, (C) CD4+CD25+Moderate and(D)CD4+CD25+High in early T2D patient.

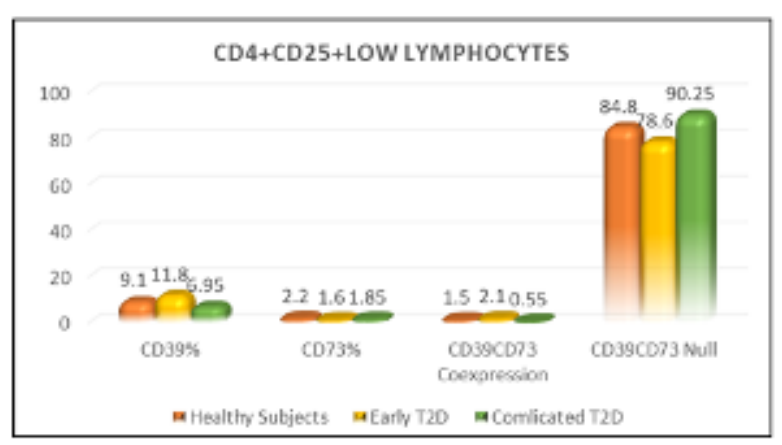

Fig. (6): Comparison of CD39 and CD73 expression on peripheral blood CD4+CD25+ Low Lymphocytes among healthy subjects, early and complicated T2D patients. 


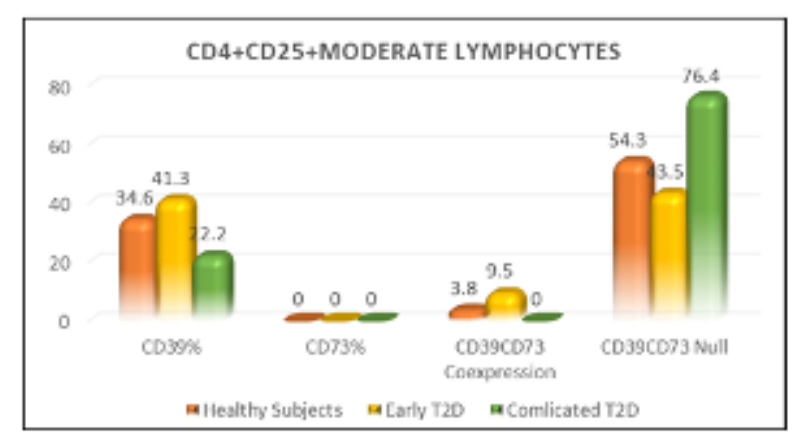

Fig. (7): Comparison of CD39 and CD73 expression on peripheral blood CD4+CD25+ Moderate Lymphocytes among healthy subjects, early and complicated T2D patients.

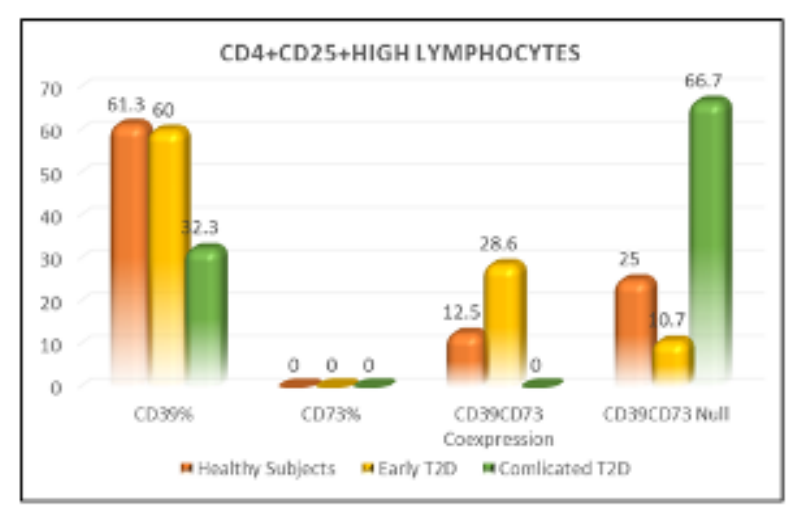

Fig. (8): Comparison of CD39 and CD73 expression on peripheral blood CD4+CD25+ High Lymphocytes among healthy subjects, early and complicated T2D patients.

A negative correlation was found between the expression of CD39 and duration of the disease, level of $\mathrm{HbA} 1 \mathrm{c}$ and triglycerides in several cell types. Moreover, CD39 expression on neutrophils was positively correlated with BMI. In addition, positive correlation was found between $\mathrm{CD} 73$ expression and level of $\mathrm{HbA1C}$ and LDL.

\section{DISCUSSION}

In our study we found that there is an increase in CD39 expression on total lymphocytes (Table 1, Fig. 2), CD4+ lymphocytes (Table 2, Fig. 3\&4), CD4+CD25+ lymphocytes subpopulations $\quad\left(\mathrm{CD} 4+\mathrm{CD} 25+{ }^{\mathrm{High}}\right.$ $\mathrm{CD} 4+\mathrm{CD} 25+{ }^{\text {Moderate }}$ and $\mathrm{CD} 4+\mathrm{CD} 25+{ }^{\mathrm{Low}}$ ) (Table 3, Fig. 5-8) in early cases of $T 2 D$ compared to complicated cases and healthy subjects. Meanwhile, CD73 showed comparable expression on these cells among early and complicated T2D patients and healthy controls.

Our findings agreed with the study by Guzman-Flores et al. ${ }^{(\boldsymbol{\sigma})}$ who also detected CD39 expression on CD4+ cells in non-complicated cases of T2D giving same results of increased expression of CD39 and decrease expression of CD73.

Garcia-Hernandez et al. ${ }^{(7)}$ also reported an increase in the level of expression and function of CD39 in early T2D patients, which represent high numbers of activated cells contributing to a lowlevel chronic inflammation specific to these patients. Thus, high expression of CD39 in peripheral blood mononuclear cells might be seen as a marker of chronically activated immune cells.

Knowing that CD39 and CD73 share in metabolizing ATP to adenosine ${ }^{(8)}$, the difference in expression between the two markers seems contradictory. However, a soluble form of human CD73 has been described ${ }^{(9)}$. Treg cells secrete exosomes expressing CD73 contributing to their suppressive activity ${ }^{(10)}$. Moreover, it was reported that CD73 is expressed more widely in the intracellular environment (11). Therefore, low levels of expression of CD73 in diabetic patients could be attributed to its presence in a secretory particle or intracellular form.

As was mentioned before, both CD39 and CD73 are expressed in Treg cells ${ }^{(8)}$, therefore we studied the expression of these markers in $\mathrm{CD} 4+\mathrm{CD} 25+{ }^{\text {High }}, \quad \mathrm{CD} 4+\mathrm{CD} 25+{ }^{\text {Moderate }}$ and $\mathrm{CD} 4+\mathrm{CD} 25+{ }^{\text {Low }}$ lymphocytes. High expression of CD39 coupled with low expression of CD73 was found, in the three Tregs lymphocyte subpopulations, among the early, non-complicated diabetic patients in contrast to the healthy subjects who were found to have less degree of expression of CD39 on these cells. It was previously reported that $\mathrm{CD} 4+\mathrm{CD} 25+{ }^{\text {High }} \mathrm{CD} 39+$ cells have a regulatory phenotype ${ }^{(11)}$.

These results were in accordance with Guzman-Flores et al. (6) who also reported higher expression of CD39 coupled with low expression of CD73 on the three Tregs lymphocyte subsets in noncomplicated diabetic patients in contrast to healthy subjects.

Taking in consideration the role of adenosine as an anti-inflammatory mediator, it can be considered as a protective factor in the progression of diabetes and its complications. So in order to evaluate this role we compared the expression of CD39 and CD73 in complicated long standing T2D to early T2D and healthy subjects. We found that there was a decrease in the degree of expression of CD39 and CD73 in the complicated cases in comparison to early cases and healthy subjects on total lymphocytes (Table 1, Fig. 2), CD4+ lymphocytes 
(Table 2, Fig. 3 \& 4), CD4+CD25+ lymphocytes subpopulations (Table 3, Fig. 5-8).

These results can be verified and supported by various animal experiments highlighting the protective role of adenosine, which was cancelled upon using an antagonist or by genetic deletion.

For example, an experiment done on murine model of thromboplastin-induced thromboembolism revealed decrease in mortality upon treatment of the murine model with CD39 in liposomal formulations (12). In addition, transgenic expression of CD39 reduced infarct size in a swine model of myocardial ischemia/reperfusion injury ${ }^{(\mathbf{1 3})}$.

The equilibrium of extracellular ATP metabolism on the endothelium controls the inflammatory state of the surrounding environment (14). This decrease ADP concentration (a platelet activator) and produce adenosine, a powerful, locally acting inhibitor of platelet reactivity ${ }^{(15)}$. The action of CD39 in the metabolism of ATP and ADP into AMP make it a major promoter of platelet inhibition and significantly reduce or even abolish platelet aggregation and recruitment ${ }^{(\mathbf{1 6})}$.

To reveal the functional role for CD73 during diabetic nephropathy, a study was conducted on CD73- mice by inducing diabetes mellitus with streptozotocin treatment. In CD73-mice, a more severe degree of renal dysfunction (as evaluated by measurements of glomerular filtration rate and albuminuria), low transcript levels of renal nephrin (a protein essential for the appropriate function of the renal filtration barrier) ${ }^{(17)}$, increase of inflammatory markers and more severe histologic tissue injury was found (18). Taking together, the important protective role of extracellular adenosine during diabetic nephropathy is highlighted.

In our study when we correlated the levels of CD39 and CD73 expression and various anthropometric and biochemical parameters. We found that there was a negative correlation between CD39 expression level and duration of the disease, level of HbAlc and triglyceride. This support the concept that with longstanding diabetes and poor control the protective role of adenosine produced from $\mathrm{CD} 39 / \mathrm{CD} 73$ axis is decreased favoring the progression of diabetic complications. This correlation became positive when it was tested against BMI, which was in accordance with the study done by Guzman-Flores et al. ${ }^{(6)}$ who found positive correlation between level of CD39 expression and age and BMI.

Future challenges will include the translation of these findings from bench to bedside. Particularly, it will be critical to move forward with $\mathrm{A} 2 \mathrm{~b}$ agonist treatment in human disease conditions. The limitation to the therapeutic use of adenosine and its agonists is due to its systemic side effects, such as hypotension, bradycardia, and sedation ${ }^{\left({ }^{(19)}\right.}$.

\section{CONCLUSION}

The increased expression of CD39 coupled with decreased expression of CD73 on peripheral blood lymphocytes (total lymphocytes, CD4+ lymphocytes and CD4+CD25+HighTregs) in early non-complicated cases of T2D indicates a state of low-level chronic inflammation owing to their role in production of adenosine; a potent anti-inflammatory mediator. This is reversed when CD39 and CD73 expression in peripheral blood leucocytes is assessed in complicated T2D patients, which emphasise their role in protecting the patients from diabetic complications.

\section{ACKNOWLEDGEMENT}

\section{REFERENCES}

1. Rother KI (2007): General aspects of diabetes mellitus. N. Englg. J. Med., 356 (15): 1499-1501.

2. Eberhart MS, Ogden C, Engelgau $M$ et al. (2004): Prevalence of overweight and obesity among adults with diagnosed diabetes-United States, 1988-1994 and 1999-2002. Biochem. Biophys. Res. Commun., 3(300): 674-678.

3. Sacks DB and McDonald JM (2006): The pathogenesis of type II diabetes mellitus. A polygenic disease. Am. J. Clin. Pathol., 115 (2):149-156.

4. Esser N, Legrand-Poels S, Piette $J$ et al. (2014): Inflammation as a link between obesity, metabolic syndrome and type 2 diabetes. Diabetes Res. Clin. Pract., 105:141-150.

5. Kaku H, Cheng KF, Al-Abed $Y$ and Rothstein TL (2014): A Novel Mechanism of B Cell-Mediated Immune Suppression through CD73 Expression and Adenosine Production.J. Immunol., 193(12):5904-5913

6. Guzman-Flores JM, Cortez-Espinosa N, Cortez-Garcia JD et al. (2015): Expression of CD73 and A2A receptors in cells from subjects with obesity and Type 2 Diabetes Mellitus. Immunobiology, 220(8):976-984. 
7. Garcia-Hernandez MH, Portales-Cervantes L, Cortez-Espinosa $N$ et al. (2011): Expression and function of $\mathrm{P} 2 \mathrm{X}(7)$ receptor and CD39/Entpd1 in patients with type 2 diabetes and their association with biochemical parameters. Cell Immunol., 269:135-143.

8. Deaglio S, Dwyer KM, Gao W et al. (2007): Adenosine generation catalyzed by CD39 and CD73 expressed on regulatory $\mathrm{T}$ cells mediates immune suppression. Journal of Experimental Medicine, 204(6):1257-1265.

9. Heuts DP, Weissenborn MJ, Olkhov RV et al. (2012): Crystal structure of a soluble form of human CD73 with ecto-5'-nucleotidase activity. Chembiochem., 13:2384-2391.

10. Smyth LA, Ratnasothy K, Tsang JY et al. (2013): CD73 expression on extracellular vesicles derived from CD4+ CD25+ Foxp3+ Tcells contributes to their regulatory function. Eur. J. Immunol., 43:2430-2440.

11. Mandapathil M, Hilldorfer B, Szczepanski MJ et al. (2010): Generation and accumulation of immunosuppressive adenosine by human CD4+CD25high FOXP3+ regulatory T cells. J. Biol. Chem., 285:7176-7186.

12. Haller CA, Cui W, Wen $J$ et al. (2006): Reconstitution of CD39 in liposomes amplifies nucle-oside triphosphate diphosphohydrolase activity and restores thromboregulatory properties. J. Vasc. Surg., 43(4):816-823.

13. Wheeler DG, Joseph ME, Mahamud SD et al. (2012): Transgenic swine: expression of human CD39 protects against myocardial injury. J. Mol. Cell Cardiol., 52(5):958-961.
14. Bours MJ, Swennen EL, Di Virgilio F et al. (2006): Adenosine 5'-triphosphate and adenosine as endogenous signaling molecules in immunity and inflammation. Pharmaco. Ther., 112(2):358-404.

15. Fuentes E, Badimon $L$, Caballero $J$ et al. (2014): Protective mechanisms of adenosine 5'monophosphate in platelet activation and thrombus formation. Thromb. Haemost., 111(3):491-507.

16. Pulte D, Olson KE, Broekman MJ et al. (2007): CD39 activity correlates with stage and inhibits platelet reactivity in chronic lymphocytic leukemia. J. Transl. Med., 5:23.

17. Nadarajah R, Milagres $R$, Dilauro $M$ et al. (2012): Podocyte-specific overexpression of human angiotensin-converting enzyme 2 attenuates diabetic nephropathy in mice. Kidney Int., 82:292-303.

18. Tak E, Ridyard D, Kim JH et al. (2014): CD73-dependent generation of adenosine and endothelial Adora2b signaling attenuate diabetic nephropathy.J. Am. Soc. Nephrol., 25(3):547-563.

19. Williams M (1996): Challenges in developing P2 purinoceptor-based therapeutics. CIBA Found Symp., 198:309-321. 